\title{
On Hierarchical Error Estimators for Time-discretized Phase Field Models
}

\author{
Carsten Gräser, Ralf Kornhuber, and Uli Sack*
}

\begin{abstract}
We suggest hierarchical a posteriori error estimators for time-discretized Allen-Cahn and Cahn-Hilliard equations with logarithmic potential and investigate their robustness numerically. We observe that the associated effectivity ratios seem to saturate for decreasing mesh size and are almost independent of the temperature.
\end{abstract}

\section{Introduction}

Hierarchical a posteriori error estimators are based on the extension of the given finite element space $\mathcal{S}$ by an incremental space $\mathcal{V}$. After discretization of the actual defect problem with respect to the extended space $\mathcal{Q}=\mathcal{S}+\mathcal{V}$, hierarchical preconditioning and subsequent localization give rise to local defect problems associated with low-dimensional subspaces of $\mathcal{V}$. The resulting local contributions to the desired global error estimate are often used as error indicators in an adaptive refinement process. We refer to the pioneering work of Zienkiewicz et al. [23] and Deuflhard et al. [10] or to the monograph of Ainsworth and Oden [1].

Local lower bounds by hierarchical error estimators typically come without unknown constants, e.g., for linear self-adjoint problems. In this sense, hierarchical error estimators are properly scaled by construction. In early papers, upper bounds are often derived from the so-called saturation assumption that the extended space $\mathcal{Q}$ provides a more accurate approximation than $\mathcal{S}$. It turned out later that local equivalence to residual estimators provides upper bounds up to data oscillation and, conversely, that small data oscillation implies the saturation assumption [7, 12]. For a direct proof based on local $L^{2}$-projections we refer to [24].

Another attractive feature of hierarchical error estimators is their intriguing simplicity, particularly as applied to nonlinear, non-smooth problems $[2,17,19,20$,

\footnotetext{
* Freie Universität Berlin, Mathematical Institute (http://numerik.mi.fu-berlin.de/). This work was supported by the DFG Research Center MATHEON.
} 
$21,22,24]$. In this numerical study, we consider hierarchical error estimators for semi-linear elliptic problems as arising from the time discretization of Allen-Cahn and Cahn-Hilliard equations. While previous work concentrates on quartic shallow quench approximations $[4,13,18]$ or on heuristic strategies for obstacle potentials [5] we consider the logarithmic potential here. In particular, we investigate the robustness of the effectivity ratios as temperature is approaching the deep quench limit. In our numerical experiments, we found that both for Allen-Cahn- and Cahn-Hilliard-type problems the associated effectivity ratios seem to saturate with decreasing mesh size and are hardly influenced by temperature. Moreover, the local contributions to the global estimator were used successfully for adaptive refinement.

\section{Hierarchical Error Estimators}

In this section, we derive hierarchical error estimators in an abstract setting. Special cases will be considered later on. For ease of presentation, we assume that all occurring problems and subproblems are uniquely solvable. Let $H$ denote a Hilbert space with the norm $\|\cdot\|_{H}$. We consider the variational inequality

$$
u \in H: \quad a(u, v-u)+\phi(v)-\phi(u) \geq \ell(v-u) \quad \forall v \in H
$$

with $a(\cdot, \cdot), \phi: H \rightarrow \mathbb{R} \cup\{+\infty\}$, and $\ell$ denoting a symmetric bilinear form, a convex functional, and a bounded linear functional on $H$, respectively. The additional conditions that $a(\cdot, \cdot)$ is $H$-elliptic and that $\phi$ is lower semi-continuous and proper are sufficient but not necessary to ensure existence and uniqueness [14]. Let $\mathcal{S}$ denote a finite-dimensional subspace of $H$ and let the symmetric bilinear form $a_{\mathcal{S}}(\cdot, \cdot)$ and the functional $\phi_{\mathcal{S}}: \mathcal{S} \rightarrow \mathbb{R} \cup\{+\infty\}$ be approximations of $a(\cdot, \cdot)$ and $\phi$ on $\mathcal{S}$, respectively, e.g., by numerical quadrature like mass lumping. Then the associated Ritz-Galerkin discretization reads

$$
u_{\mathcal{S}} \in \mathcal{S}: \quad a_{\mathcal{S}}\left(u_{\mathcal{S}}, v-u_{\mathcal{S}}\right)+\phi_{\mathcal{S}}(v)-\phi_{\mathcal{S}}\left(u_{\mathcal{S}}\right) \geq \ell\left(v-u_{\mathcal{S}}\right) \quad \forall v \in \mathcal{S} .
$$

We want to derive a posteriori estimates of the error $\left\|u-u_{\mathcal{S}}\right\|_{H}$. To this end, we consider the defect problem

$$
e \in H: \quad a(e, v-e)+\psi(v)-\psi(e) \geq r(v-e) \quad \forall v \in H
$$

involving the shifted nonlinearity $\psi$ and the residual $r$, defined by

$$
\psi(v)=\phi\left(u_{\mathcal{S}}+v\right), \quad r(v)=\ell(v)-a\left(u_{\mathcal{S}}, v\right), \quad v \in H,
$$

respectively. Obviously, $u=u_{\mathcal{S}}+e$. To approximate (3), we select an incremental space $\mathcal{V} \subset H$ with the property $\mathcal{V} \cap \mathcal{S}=\{0\}$ and consider the hierarchical extension

$$
\mathcal{Q}=\mathcal{S} \oplus \mathcal{V}
$$


of $\mathcal{S}$. The subspace $\mathcal{Q} \subset H$ is equipped with the discrete norm $\|\cdot\|_{\mathcal{Q}}$ which intentionally is an equivalent approximation of $\|\cdot\|_{H}$. The associated discretized defect problem is given by

$$
e_{\mathcal{Q}} \in \mathcal{Q}: \quad a_{\mathcal{Q}}\left(e_{\mathcal{Q}}, v-e_{\mathcal{Q}}\right)+\psi_{\mathcal{Q}}(v)-\psi_{\mathcal{Q}}\left(e_{\mathcal{Q}}\right) \geq r\left(v-e_{\mathcal{Q}}\right) \quad \forall v \in \mathcal{Q}
$$

with $a_{\mathcal{Q}}(\cdot, \cdot)$ and $\psi_{\mathcal{Q}}: \mathcal{Q} \rightarrow \mathbb{R} \cup\{+\infty\}$ denoting approximations of $a(\cdot, \cdot)$ and $\psi$. In order to avoid the computational effort for the computation of $e_{\mathcal{Q}}$, we now modify (4) in a way that allows for a decomposition into a number of independent, lowdimensional subproblems. This modification is based on the assumption that the given nonlinearity is local in the sense that there is a direct splitting

$$
\mathcal{V}=\mathcal{V}_{1} \oplus \cdots \oplus \mathcal{V}_{m}
$$

of $\mathcal{V}$ into low-dimensional subspaces $\mathcal{V}_{i}$ such that for $v \in \mathcal{V}$ the representation

$$
\psi_{\mathcal{Q}}(v)=\sum_{i=1}^{m} \Psi_{i}\left(v_{i}\right)
$$

holds with certain convex functionals $\Psi_{i}: \mathcal{V}_{i} \rightarrow \mathbb{R} \cup\{+\infty\}$ and the uniquely determined decomposition $v=\sum v_{i}, v_{i} \in \mathcal{V}_{i}$. Then, in the first step, we replace the bilinear form $a(\cdot, \cdot)$ by the hierarchical preconditioner

$$
b(v, w)=a_{\mathcal{Q}}\left(v_{\mathcal{S}}, w_{\mathcal{S}}\right)+\sum_{i=1}^{m} a_{\mathcal{Q}}\left(v_{i}, w_{i}\right), \quad v, w \in \mathcal{Q},
$$

based on the uniquely determined decompositions of $v=v_{\mathcal{S}}+v_{\mathcal{V}} \in \mathcal{Q}$ into $v_{\mathcal{S}} \in \mathcal{S}$, $v_{\mathcal{V}} \in \mathcal{V}$ and of $v_{\mathcal{V}}=\sum v_{i}, w_{\mathcal{V}}=\sum w_{i}$ into $v_{i}, w_{i} \in \mathcal{V}_{i}$. It can be shown under certain conditions [19] that the solution $\tilde{e}_{\mathcal{Q}}$ of the resulting preconditioned defect problem provides an efficient and reliable error estimate $b\left(\tilde{e}_{\mathcal{Q}}, \tilde{e}_{\mathcal{Q}}\right)^{1 / 2}$. However, the exact evaluation of $\tilde{e}_{\mathcal{Q}}=\tilde{e}_{\mathcal{S}}+\tilde{e}_{\mathcal{V}}$ is still too costly: In contrast to linear situations, we cannot expect $\tilde{e}_{\mathcal{S}}=0$, because $\tilde{e}_{\mathcal{S}} \in \mathcal{S}$ and $\tilde{e}_{\mathcal{V}} \in \mathcal{V}$ are still coupled with respect to the nonlinearity $\psi_{\mathcal{Q}}(v)=\psi_{\mathcal{Q}}\left(v_{\mathcal{S}}+v_{\mathcal{V}}\right)$. As a remedy, we simply assume that the low-frequency part $\tilde{e}_{\mathcal{S}}$ of our error estimate can be neglected. In this way, we finally obtain the localized defect problem

$$
e_{\mathcal{V}} \in \mathcal{V}: \quad b\left(e_{\mathcal{V}}, \mathcal{v}-e_{\mathcal{V}}\right)+\psi_{\mathcal{Q}}(v)-\psi_{\mathcal{Q}}\left(e_{\mathcal{V}}\right) \geq r\left(v-e_{\mathcal{V}}\right) \quad \forall v \in \mathcal{V} .
$$

It has been shown for obstacle problems that reliability might get lost by this localization step but can be reestablished by a suitable higher order term [20]. Exploiting assumption (5), the evaluation of $e_{\mathcal{V}}=\sum e_{i}$ amounts to the solution of $m$ independent subproblems

$$
e_{i} \in \mathcal{V}_{i}: \quad a_{\mathcal{Q}}\left(e_{i}, v-e_{i}\right)+\Psi_{i}(v)-\Psi_{i}\left(e_{i}\right) \geq r\left(v-e_{i}\right) \quad \forall v \in \mathcal{V}_{i}
$$

The quantity 


$$
\eta=\left(\sum_{i=1}^{m}\left\|e_{i}\right\|_{\mathcal{Q}}^{2}\right)^{1 / 2}
$$

is our hierarchical error estimator. If $a_{\mathcal{Q}}(\cdot, \cdot)$ is $\mathcal{Q}$-elliptic and $\mathcal{Q}$ is equipped with the energy norm $\|\cdot\|_{\mathcal{Q}}=a_{\mathcal{Q}}(\cdot, \cdot)^{1 / 2}$, then (8) takes the form $\eta=b\left(e_{\mathcal{V}}, e_{\mathcal{V}}\right)^{1 / 2}$.

\section{Allen-Cahn Equations}

Implicit time discretization of the Allen-Cahn equation with logarithmic potential (see, e.g. [9, Section 7] for an overview) gives rise to spatial problems of the form (1) with $H=H^{1}(\Omega)$ equipped with the energy norm induced by the bilinear form

$$
a(v, w)=\gamma(v, w)_{L^{2}(\Omega)}+\tau(\nabla v, \nabla w)_{L^{2}(\Omega)}, \quad \gamma=1-\tau \theta_{c} / \varepsilon^{2}
$$

the right hand side $\ell(v)=\left(u_{0}, v\right)_{L^{2}(\Omega)}$, and the convex, lower semi-continuous, proper functional $\phi(v)=\int_{\Omega} \Phi^{\theta}(v) d x$ with

$$
\Phi^{\theta}(v)=\left\{\begin{array}{cl}
\frac{\tau}{2 \varepsilon^{2}} \theta((1+v) \log (1+v)+(1-v) \log (1-v)), & \text { if } \theta>0 \\
\chi_{[-1,1]}(v), & \text { if } \theta=0
\end{array} .\right.
$$

Here, $\Omega \subset \mathbb{R}^{2}$ denotes a polygonal domain, $\varepsilon>0$ is an interface parameter, $\theta \geq 0$ and $\theta_{c}>0$ stand for the temperature and the critical temperature, respectively, $\chi_{[-1,1]}$ is the characteristic function of $[-1,1], u_{0} \in L^{2}(\Omega)$ is an approximation from the preceding time step, and $\tau>0$ is the time step size. We assume $\gamma>0$ or, equivalently, $\tau<\varepsilon^{2} / \theta_{c}$ so that $a(\cdot, \cdot)$ is $H$-elliptic. With these definitions, (1) can be rewritten as a semi-linear elliptic problem for positive temperature $\theta>0$ and as an elliptic obstacle problem for $\theta=0$.

Let $\mathcal{S}=\mathcal{S}_{h}$ denote the space of piecewise linear finite elements with respect to a regular triangulation $\mathcal{T}_{h}$ with mesh size $h$ and interior vertices $\mathcal{N}_{h}$. Then, $a_{\mathcal{S}}(\cdot, \cdot)=a_{h}(\cdot, \cdot)$ is defined by replacing $(v, w)_{L^{2}(\Omega)}$ with the lumped $L^{2}$-scalar product $\langle v, w\rangle_{\mathcal{S}}=\int_{\Omega} I_{h}(v w) d x$, where $I_{h}: C(\bar{\Omega}) \rightarrow \mathcal{S}_{h}$ denotes nodal interpolation. Similarly, we set $\phi_{\mathcal{S}}(v)=\phi_{h}(v)=\int_{\Omega} I_{h}\left(\Phi^{\theta}(v)\right) d x$. Connecting the midpoints $p_{i}$ of the edges of all triangles $t \in \mathcal{T}_{h}$, we obtain the uniformly refined triangulation $\mathcal{T}_{h / 2}$ with interior vertices $\mathcal{N}_{h / 2}$. The local incremental spaces $\mathcal{V}_{i}=\operatorname{span}\left\{\mu_{i}\right\}$ are spanned by the piecewise linear edge bubble functions satisfying $\mu_{i}\left(p_{i}\right)=1$ and vanishing on all other vertices $p \in \mathcal{N}_{h / 2}$. This choice leads to $\mathcal{Q}=\mathcal{S}_{h / 2}$. It is motivated by the lack of stability of piecewise quadratic approximations for obstacle problems [14, 20]. We select the discrete $\mathcal{Q}$-elliptic bilinear form $a_{\mathcal{Q}}(\cdot, \cdot)=a_{h / 2}(\cdot, \cdot)$ with the associated energy norm $\|\cdot\|_{\mathcal{Q}}=a_{h / 2}(\cdot, \cdot)^{1 / 2}$. Then the locality condition (5) is satisfied with $\Psi_{i}(v)=\Phi^{\theta}\left(\left(u_{\mathcal{S}}+v\right)\left(p_{i}\right)\right) \int_{\Omega} \mu_{i} d x, v \in \mathcal{V}_{i}$. For obstacle problems, i.e. for $\theta=0$, the resulting error estimator (8) was proposed in [17] and later analyzed in [20, 22, 24]. Here we concentrate on $\theta>0$ and investigate robustness for $\theta \rightarrow 0$. 
In our numerical experiments, we consider the first spatial problem of the semidiscrete Allen-Cahn equation with parameters $\varepsilon=2 \cdot 10^{-2}, \theta_{c}=1.0$, time step size $\tau=10^{-4}$, and the initial condition $u_{0}$ as depicted in the left picture of Figure 3 . We first compare the a posteriori error estimator with the 'exact' error for a fixed temperature $\theta=0.1$ and a sequence of triangulations $\mathcal{T}_{h_{j}}$ with decreasing mesh size $h_{j}$. The triangulations $\mathcal{T}_{j}=\mathcal{T}_{h_{j}}$ are obtained by $j=1, \ldots, 9$ uniform refinements of the initial triangulation $\mathcal{T}_{0}$ which is a partition of $\Omega=(-1,1) \times(-1,1)$ into two congruent triangles. The 'exact' error $\tilde{e}_{j}=\left\|\tilde{u}-u_{j}\right\|_{\tilde{H}}$ is obtained by approximating $H$ with $\tilde{H}=\mathcal{S}_{11}$, i.e., by an approximation $\tilde{u}$ of $u$ based on two further uniform refinement steps. The left picture in Figure 1 shows $\eta_{j}$ and $\tilde{e}_{j}$ over the number of unknowns. We observe asymptotic first order convergence and a good agreement of $\eta_{j}$ and $\tilde{e}_{j}$. More precisely, the effectivity ratios $\eta_{j} / \tilde{e}_{j}$ seem to saturate at about 0.9 (Figure 1 right). In our next experiment, we fix the mesh $\mathcal{T}_{9}$ and vary the temperature
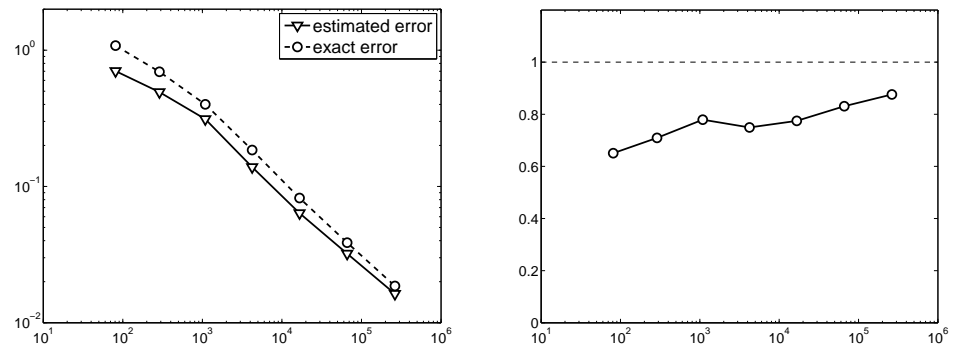

Fig. 1 Estimated and 'exact' error (left) and effectivity ratio (right) over number of unknowns.

$\theta$. The left picture in Figure 2 shows that the effectivity ratios are hardly affected by the transition from a shallow to a deep quench and even seem to converge in the deep quench limit. In the last experiment, we use the edge-oriented local error indicators
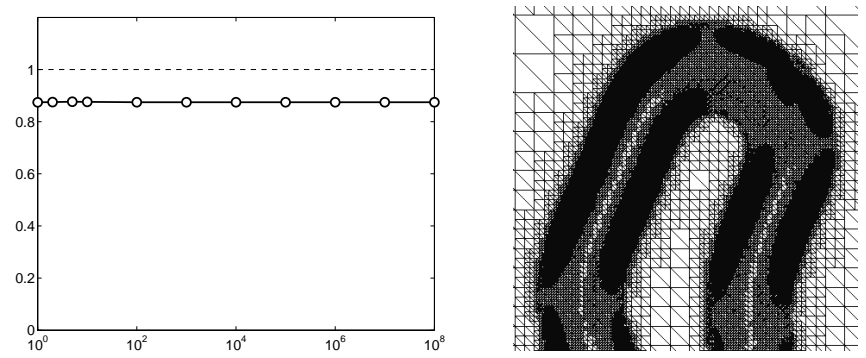

Fig. 2 Effectivity ratios over inverse temperature (left) and detail of adaptively refined mesh (right)

$\left\|e_{i}\right\|_{\mathcal{Q}}$ occurring in the global estimate (8) and a classical marking strategy [11] for adaptive mesh refinement. Figure 3 illustrates that refinement nicely follows the diffuse interface. Moreover, the zoom in Figure 2 shows that refinement concentrates 


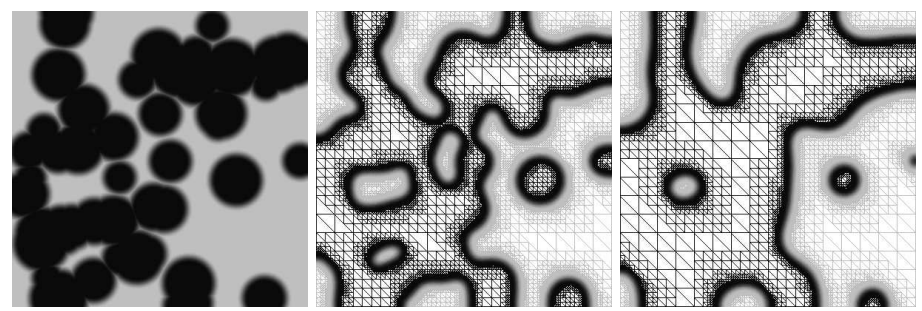

Fig. 3 Initial condition and approximations at time $20 \tau, 100 \tau$

on the strong variation of the solution at the boundary of the diffuse interface and not on the interior where steep gradients are resolved sufficiently well. Finally, note that optimal order of convergence is preserved by adaptivity.

\section{Cahn-Hilliard equations}

Semi-implicit time discretization $[6,16]$ of the Cahn-Hilliard equation with logarithmic potential leads to spatial problems of the form (1) with $H=H^{1}(\Omega) \times H^{1}(\Omega)$ equipped with norm

$$
\left\|\left(v_{1}, v_{2}\right)\right\|_{H}^{2}=\varepsilon^{2}\left(\left\|\nabla v_{1}\right\|_{L^{2}(\Omega)}^{2}+\left(v_{1}, 1\right)_{L^{2}(\Omega)}^{2}\right)+\tau\left(\left\|\nabla v_{2}\right\|_{L^{2}(\Omega)}^{2}+\left\|v_{2}\right\|_{L^{2}(\Omega)}^{2}\right),
$$

the indefinite bilinear form

$$
\begin{array}{r}
a(v, w)=\varepsilon^{2}\left(\left(\nabla v_{1}, \nabla w_{1}\right)_{L^{2}(\Omega)}+\left(v_{1}, 1\right)_{L^{2}(\Omega)}\left(w_{1}, 1\right)_{L^{2}(\Omega)}\right)-\left(v_{2}, w_{1}\right)_{L^{2}(\Omega)} \\
-\left(v_{1}, w_{2}\right)_{L^{2}(\Omega)}-\tau\left(\nabla v_{2}, \nabla w_{2}\right)_{L^{2}(\Omega)},
\end{array}
$$

the right hand side $\ell(v)=\left(u_{0}, v_{1}-v_{2}\right)_{L^{2}(\Omega)}+\varepsilon^{2}\left(u_{0}, 1\right)_{L^{2}(\Omega)}\left(v_{1}, 1\right)_{L^{2}(\Omega)}$ and the convex functional $\phi(v)=\int_{\Omega} \Phi^{\theta}\left(v_{1}\right) d x$ with $\Phi^{\theta}$ defined in (9) for temperature $\theta \geq 0$. Here, $\varepsilon$ is an interface parameter, $\tau$ is the time step size and $u_{0}$ is an approximation from the preceding time step. Utilizing the notation of Section 3, the approximation (2) is based on $\mathcal{S}=\mathcal{S}_{h} \times \mathcal{S}_{h}$, and on $a_{\mathcal{S}}(\cdot, \cdot)$ and $\phi_{\mathcal{S}}$ as obtained by mass lumping. Existence, uniqueness, and convergence results have been established in [6] for the double obstacle case $\theta=0$ and in [3, 8] for $\theta>0$. Fast solvers for the resulting algebraic problems are described in [15]. The components $u_{1}$ and $u_{2}$ of the solution $u=\left(u_{1}, u_{2}\right)$ are often called order parameter and chemical potential, respectively. Similar to Section 3, we select the incremental spaces $\mathcal{V}_{i}=\operatorname{span}\left\{\left(\mu_{i}, 0\right),\left(0, \mu_{i}\right)\right\}$ providing $\mathcal{Q}=\mathcal{S}_{h / 2} \times \mathcal{S}_{h / 2}$. Again (5) is satisfied with $\Psi_{i}(v)=\Phi^{\theta}\left(\left(u_{\mathcal{S}, 1}+v_{1}\right)\left(p_{i}\right)\right) \int_{\Omega} \mu_{i} d x, v \in \mathcal{V}_{i}$. In this setting the localized defect problem (6) admits a unique solution. The discrete norm $\|\cdot\|_{\mathcal{Q}}$ is obtained by (spectrally equivalent) mass lumping of the zero order terms in $\|\cdot\|_{H}$. 

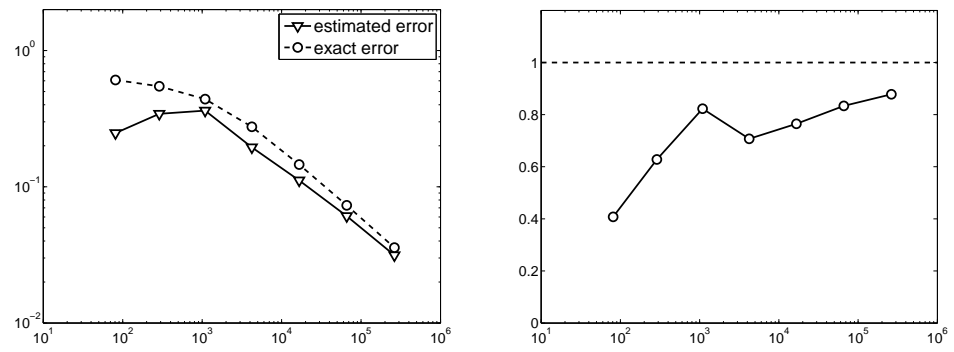

Fig. 4 Estimated and 'exact' error (left) and effectivity ratios over number of unknowns.

We consider the first spatial problem of the semi-discrete Cahn-Hilliard equation with parameters, time step size, and initial condition given in Section 3. In our numerical experiments, we proceed in complete analogy to the previous section. We begin with a comparison of the error estimators $\eta_{j}$ with an 'exact' error $\tilde{e}_{j}$ for fixed

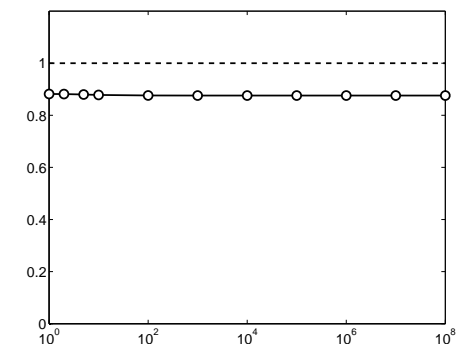

Fig. 5 Effectivity ratios over inverse temperature.

temperature $\theta=0.1$ and decreasing mesh size $h_{j}$. Figure 4 shows optimal order of convergence and asymptotic saturation of the effectivity ratios $\eta_{j} / \tilde{e}_{j}$ at about 0.9 . For fixed mesh $\mathcal{T}_{9}$ effectivity is hardly affected by strongly varying temperature $\theta$ as

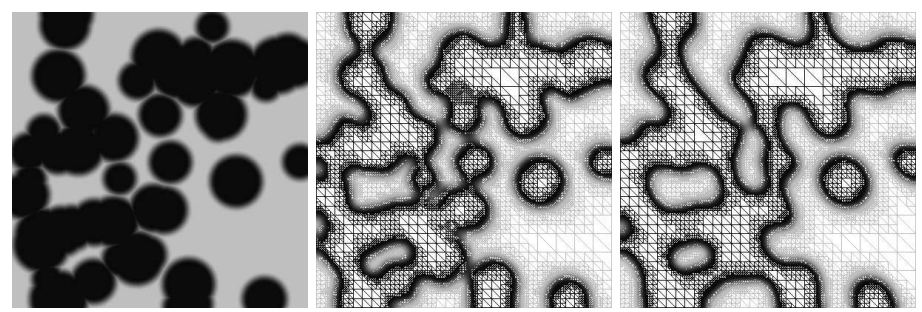

Fig. 6 Initial condition and approximations at time $20 \tau, 100 \tau$

depicted in Figure 5. Adaptive mesh refinement based on the local error indicators $\left\|e_{i}\right\|_{\mathcal{Q}}$ nicely captures strong variation of the order parameter as illustrated by Fig- 
ure 6. Strong variation of the chemical potential as occurring, e.g., after topological changes, is also reflected by adaptive refinement. Finally, it turned out that optimal order of convergence is preserved by adaptivity.

\section{References}

1. M. Ainsworth and J.T. Oden. A posteriori error estimation in FE analysis. Wiley, 2000.

2. R.E. Bank and R.K. Smith. A posteriori error estimates based on hierarchical bases. SIAM J. Numer. Anal., 30:921-935, 1993.

3. J. W. Barrett and J. F. Blowey. Finite element approximation of an Allen-Cahn/Cahn-Hilliard system. IMA J. Numer. Anal., 22(1):11-71, 2002.

4. S. Bartels and R. Müller. Optimal and robust a posteriori error estimates in $L^{\infty}\left(L^{2}\right)$ for the approximation of Allen-Cahn equations past singularities. Preprint, Uni Bonn, 2009.

5. L. Baňas and R. Nürnberg. A posteriori estimates for the Cahn-Hilliard equation with obstacle free energy. Math. Model. Numer. Anal., 43:1003-1026, 2009.

6. J.F. Blowey and C.M. Elliott. The Cahn-Hilliard gradient theory for phase separation with non-smooth free energy II: Numerical analysis. Euro. J. Appl. Math., 3:147-179, 1992.

7. F.A. Bornemann, B. Erdmann, and R. Kornhuber. A posteriori error estimates for elliptic problems in two and three space dimensions. SIAM J. Numer. Anal., 33:1188-1204, 1996.

8. M.I.M. Copetti and C.M. Elliott. Numerical analysis of the Cahn-Hilliard equation with a logarithmic free energy. Numer. Math., pages 39-65, 1992.

9. K. Deckelnick, G. Dziuk, and C.M. Elliott. Computation of geometric partial differential equations and mean curvature flow. Acta Numer., 14:139-232, 2005.

10. P. Deuflhard, P. Leinen, and H. Yserentant. Concepts of an adaptive hierarchical finite element code. IMPACT Comput. Sci. Engrg., 1:3-35, 1989.

11. W. Dörfler. A convergent adaptive algorithm for Poisson's equation. SIAM J. Numer. Anal., 33:1106-1124, 1996.

12. W. Dörfler and R.H. Nochetto. Small data oscillation implies the saturation assumption. $\mathrm{Nu}$ mer. Math., 91:1-12, 2002.

13. X. Feng and $\mathrm{H}$. Wu. A posteriori error estimates for finite element approximations of the Cahn-Hilliard equation and the Hele-Shaw flow. J. Comput. Math., 26:767-796, 2008.

14. R. Glowinski. Numerical Methods for Nonlinear Variational Problems. Springer, 1984.

15. C. Gräser. Convex Minimization and Phase Field Models. PhD thesis, FU Berlin, To appear.

16. C. Gräser and R. Kornhuber. On preconditioned Uzawa-type iterations for a saddle point problem with inequality constraints. In O.B. Widlund and D.E. Keyes, editors, Domain Decomposition Methods in Science and Engineering XVI, pages 91 - 102. Springer, 2006.

17. R.H.W. Hoppe and R. Kornhuber. Adaptive multilevel-methods for obstacle problems. SIAM J. Numer. Anal., 31(2):301-323, 1994.

18. D. Kessler, R. Nochetto, and A. Schmidt. A posteriori error control for the Allen-Cahn problem: circumventing Gronwal's inequality. M2AN, 38:129-142, 2004.

19. R. Kornhuber. A posteriori error estimates for elliptic variational inequalities. Comput. Math. Appl., 31:49-60, 1996.

20. R. Kornhuber and Q. Zou. Efficient and reliable hierarchical error estimates for the discretization error of elliptic obstacle problems. Preprint 519, Matheon Berlin, 2008.

21. O. Sander. Multi-dimensional coupling in a human-knee model. PhD thesis, FU Berlin, 2008.

22. K. G. Siebert and A. Veeser. A unilaterally constrained quadratic minimization with adaptive finite elements. SIAM J. Optim., 18:260-289, 2007.

23. O.C. Zienkiewicz, J.P. De S.R. Gago, and D.W. Kelly. The hierarchical concept in finite element analysis. Computers \& Structures, 16:53-65, 1983.

24. Q. Zou, A. Veeser, R. Kornhuber, and C. Gräser. Hierarchical error estimates for the energy functional in obstacle problems. Preprint 575, Matheon Berlin, 2009. 